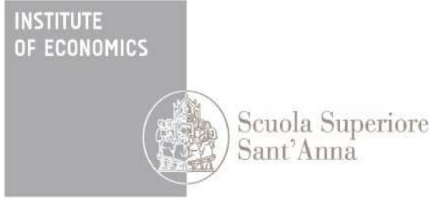

LEM | Laboratory of Economics and Management

Institute of Economics

Scuola Superiore Sant'Anna

Piazza Martiri della Libertà, 33 - 56127 Pisa, Italy ph. +3905088.33 .43$

institute.economics@sssup.it

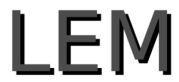

Working Paper Series

Simulating Conventions and Norms under Local Interactions and Imitation

Sebastian Ille ${ }^{\text {a }}$

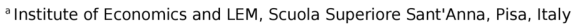




\title{
Simulating Conventions and Norms under Local Interactions and Imitation
}

\author{
SEBASTIAN ILLE \\ Laboratory of Economics and Management \\ Sant'Anna School of Advanced Studies \\ Piazza Martiri della Libertà 33 \\ 56127 Pisa (Italy) \\ sebastian.ille@sssup.it \\ http://www.lem.sssup.it/ \\ Version: 01.02.2013
}

\begin{abstract}
This paper is based on Ille 2013 Both papers analyze the same model, but in contrast, this paper does not provide an analytical solution but rather resorts to simulations. This allows the reader, who is familiar with the former article, to retrace the results more thoroughly and without the requirement of a sophisticated mathematical background. Additionally, this paper illustrates the dynamics of the setting. Focus is placed on $2 \times 2$ Nash coordination games on a two-dimensional lattice. Players imitate the most successful player in their reference group (Moore neighborhood) in the former period. Similarly individual pay-off is only defined by the current strategic choice of this reference group. We observe that the long-term convention is defined by a trade-off between risk and efficiency and that player population converges to the Pareto dominant though risk inferior convention for a broad range of pay-off configurations. In the case of two player populations, the long-term convention is defined by the equilibrium granting the highest benefit to one population. Consequently, conventions illustrate a tendency to be inegalitarian.
\end{abstract}

Keywords: Existence and Stability of Equilibria; Evolutionary Games; Behavior; Simulation Modeling.

Subject Classification: C62, C63, D73, D03, D83.

\section{Introduction}

Though oftentimes neglected in standard economic theory, the stress on the role of cultural and social characteristics in economic growth and prosperity has been been made by classic sociologists and economists more than a century ago. As Daniel Etounga-Manguelle formulated poignantly "Culture is the mother, institutions are the children" (Etounga-Manguelle in Huntington and Harrison 2004, p.135]) Institutions, however, are governed by the underlying set of norms and conventions and make up the basis for behavioral patterns and customs by which economic agents interact. These norms and conventions in turn are the stable outcomes of a large number of interactions (see also Bicchieri 2006]). "Economic reality is necessarily embedded within broader social relations, culture and institutions, and the real boundaries between the 'economy', and 'society' and 'polity' are fuzzy and unclear." 
Hodgson 1996, p.8].

In order to analyze the evolution and dynamics governing conventions and norms, this paper makes two essential assumptions: Interactions are performed only locally and each player bases his strategic choice exclusively on imitation. Global interactions are a fairly unrealistic assumption for large and dispersed player populations or if individual perception of conventions is exclusively shaped by the interaction with a reference group (parents, family, friends, colleagues, etc.). In order to localize interactions, agents are placed on a torus shaped two dimensional lattice and only interact with their surrounding neighbors. In this "spatial game" connections between players, represented by their relative spatial position, are thus of relevance.

Under more realistic conditions, the determination of a best-response strategy requires superior mental capabilities; even under simplifying assumptions that assume a bounded rationality (such as fictitious and adaptive play). The determination of a best-response strategy demands each individual to evaluate the exact expected pay-off for each strategy in his strategy set, given an anticipated strategy profile. A player requires thus both, full knowledge of his individual strategy set, and the precise associated pay-offs. In reality, individuals tend, however, to choose a strategy based on simplifying heuristics (see Page 2007). As put by Samuel Bowles: "We know that individual behavioral traits may proliferate in a population when individuals copy successful neighbors. So too may distributive norms, linguistic conventions, or individual behaviors underpinning forms of governance or systems of property rights diffuse or disappear through the emulation of the characteristics of successful groups by members of less successful groups." see Bowles 2006, p. 444]. In this paper, each player imitates his neighbor who has been most successful in the past period.

We observe that in these simplified networks, a prevailing convention will not necessarily be defined by the risk dominant outcome. The positive pay-off difference that players earn in a certain equilibrium with respect to the other equilibria, will affect its likelihood of determing the long-term social convention. In the case of local interaction and imitation, a trade-off between risk and Pareto dominance can thus be observed. This relation is a result of assortment among players with the same strategy being an evolving property in this model.

As in Ille 2013, the following section of this paper considers a symmetric $2 \times 2$ coordination game and section 3 generalizes the approach to two types of players ${ }^{a}$

Assume the following

(I.) All individuals interact on a toroid, two dimensional grid, on which they are

aThe two dimensions of interaction are not analyzed here. In this model individuals have a space which they observe, i.e. an area that defines the set of players that can be imitated, and a space which affects their benefits, i.e. the number of other surrounding players that define the individual's pay-off for each strategy in accordance to his strategic choice. For an analysis refer also to Ille 2013

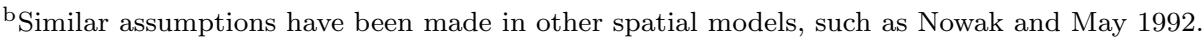


initially placed at random,

(II.) individuals only interact with their direct neighbors (Moore neighborhood),

(III.) individual pay-offs only depend on the individual's strategy and on the strategies played by his neighbors,

(IV.) each individual adopts the strategy of the neighbor with maximum pay-off in the last period; if the individual's strategy is associated with a pay-off not less than the maximum pay-off, he will keep his strategy,

(V.) all players update synchronously and once in each period,

(VI.) updating is deterministic (no mutations) and the outcome of the game is only defined by the initial conditions and distribution, and the pay-off matrix.

These assumptions will keep the analysis as simple as possible. In this paper, simulations are performed both to support results of Ille 2013 and to visualize dynamics.

\section{Symmetric pay-offs}

In this section assume that the pay-offs for two strategies $s(i)=A, B$ of player $i$ are given by a symmetric pay-off matrix with a single player type, i.e. the population is homogeneous and exists only of one type. It is thus irrelevant, whether an individual plays as row or column:

$$
\begin{aligned}
& \text { A } B \\
& \begin{array}{l}
A \\
B
\end{array}\left(\begin{array}{cc}
a, a & b, c \\
c, b & d, d
\end{array}\right)
\end{aligned}
$$

with $a>c$ and $d>b$.

Though the following analysis is local, it enables us to predict the global evolution based on the given pay-off configuration. The Pareto superior strategy is defined by the Pareto superior equilibrium. The following results are a direct consequence:

(1) In the case, where a player chooses the Pareto dominant strategy, i.e. the strategy defined by the largest value on the pay-off matrix's main diagonal, his pay-off increases with the number of neighboring players choosing the same strategy. The maximum pay-off for this strategy is obtained by individuals only surrounded by players of the same strategy. This also holds for the Pareto inferior strategy, if the matrix's main diagonal pay-off values are strictly greater than the off-diagonal values.

(2) Any interior individual, only surrounded by players of the same strategy, has never an incentive to switch, since all players in his neighborhood play the same strategy. Transitions can only occur at borders of clusters.

(3) If an individual, who is completely surrounded by players of his own strategy, plays the Pareto dominant strategy, pay-off is maximal and none of his neighbors will switch to the Pareto inferior strategy.

In order for two equilibria to be risk equivalent, it must hold that $a-c=d-b$. 
Pay-offs can be thus defined as $d=a+\rho$ and $b=c+\rho$, given that the "pay-off premium" $\rho$. For $\rho>0$ convention all $B$ (denoted by $h_{B}$ ) Pareto dominates the conventions in which all players choose $A$ (denoted by $h_{A}$ ).

From Ille 2013, we have the following definition:

Definition 1. A cluster of size $r$ is defined as a set of neighboring players, in which at least one player has $r-1$ other players with the same strategy in his neighborhood.

See figure 1 for an example: a.) and b.) show two clusters of size 4 , whereas c.) shows a cluster of size 5 .

Fig. 1. Examples for clusters of size 4 and 5

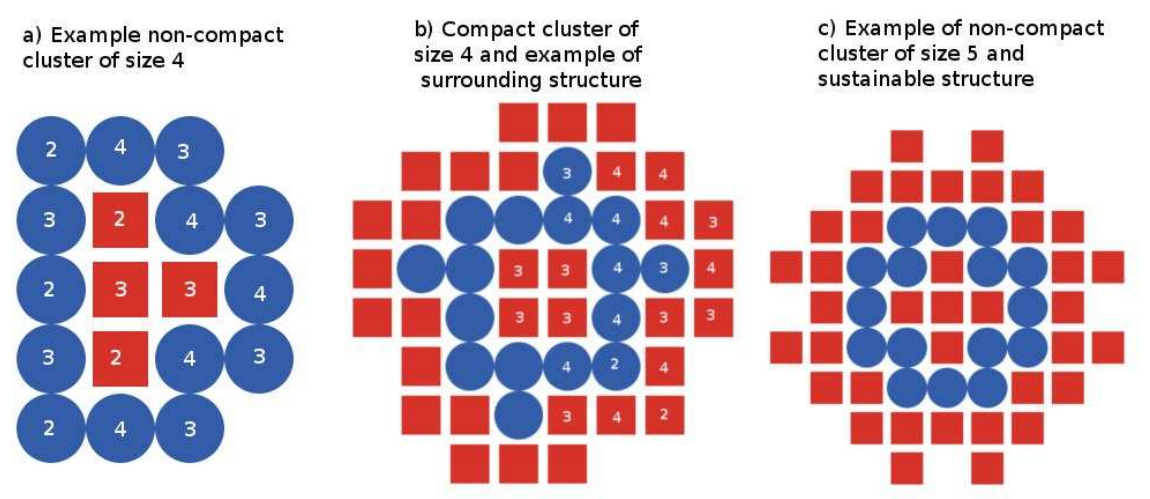

From Ille 2013 we have the following proposition:

Proposition 1. Given a pay-off matrix as in 1 with two risk equivalent pure Nash equilibria, for any $a, b, c, d$ as long as they satisfy $a>c$ and $d>b$, a population, whose convention is defined by the Pareto inferior strategy $A$, is successfully invaded by a minimal cluster of size $r$, choosing the Pareto dominant strategy $B$, if the payoff premium satisfies:

$$
\begin{array}{ll}
\begin{array}{l}
\rho>3(a-c) \text { and } r \geq 4 \text { and square } \\
\rho>\quad a-c \text { and } r \geq 5
\end{array} & \text { for } a<b \\
\rho>\frac{3}{5}(a-c) \text { and } r \geq 6 & \text { for } a \geq b
\end{array}
$$

Figure 2 shows the result of a set of simulations for $d=4, b=3, c=a-1$ and $a$ going from 3.35 to 3.55 in steps of 0.01 . The values represent the proportion of individuals playing strategy $A$ in $t \in(1,50)$, where their initial share is set to $85 \%$ 
in $t=0$ Thresholds are at their expected values. The population converges to convention all $B$ for values of $a$ smaller than 3.4 and to convention all $A$ for values larger than 3.5. Stable mixed equilibria occur for intermediate values (here: one at less than $0.8 \%$, a second at $0.4 \%$ strategy $B$ players).

Fig. 2. share of strategy $A$ players; d=4, b=3, c=a-1, $a \in(3.3,3.55 ; 0.01), t \in(1,30)$

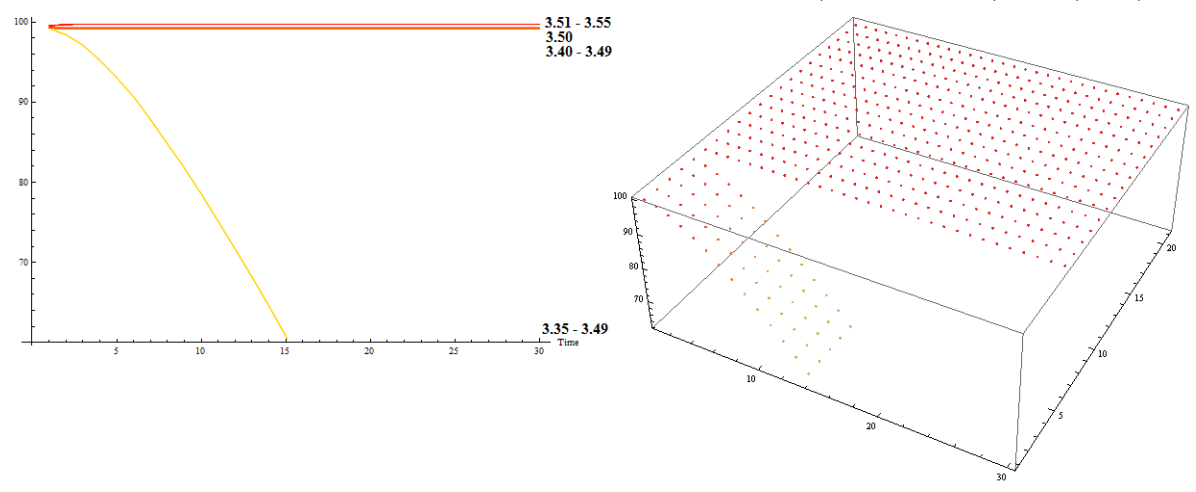

From Ille 2013 we have the following proposition

Proposition 2. Clustering is an evolving property and most clusters of at least one strategy will have a size equal to 9 after an initial period of interaction. In addition, for $b>a$ and $\rho>7(a-c)$, stable single clusters can occur, playing the Pareto inferior strategy $A$. In the case of $a>b$, clusters, playing the Pareto dominant strategy $B$, of size 6 can be stable, if $\frac{1}{2}(a-c)<\rho<\frac{3}{5} a-c$, of size 7 , if $\frac{1}{5}(a-c)<\rho<\frac{1}{3}(a-c)$, and of size 8 , if $0<\rho<\frac{1}{7}(a-c)$. Cluster of size 5 are stable iff $a=b$.

Most clusters eventually reach a size 9 , since for sizes lower than 9 clusters are required to be organized in such a way that no element inside nor outside the cluster has an incentive to change. This is geometrically impossible for a larger number of smaller clusters in a sufficiently large population. A random seeding can thus not generate a stable network. One cluster collapses and triggers the collapse of others. (For an example of how a stable population should be structured around a cluster of size 5, refer to figure 1 C.) We observe that a stable blue cluster surrounding the outer red cluster is unlikely to occur. Once the stable structure breaks apart and this red cluster changes in size and, the inner blue will do so and then also the red cross in the center.

${ }^{\mathrm{c}}$ Remember that initial seeding is random. An initial share of $15 \% \mathrm{~B}$ players generates a cluster of size 6 with positive probability for the given population size. We thus only approximate the case, in which playing strategy $A$ is a convention. 
Some or all clusters will attain a size of 9 for at least one strategy. Whether this is the case for one or both strategies depends on the initial distribution. As in Ille 2013 define

Definition 2. A balanced initial distribution defines an initial distribution of a player population, in which the average cluster size for all strategies is roughly identical after the first period of interaction. An unbalanced initial distribution defines an initial player distribution, in which average cluster sizes differ strongly among strategies after the first period of interaction, but the evolution of at least one cluster of at least size 6 occurs with certainty for any strategy after an initial sequence of interactions.

Hence, a balanced initial distribution defines the case, in which all players initially chose one of both strategies completely at random, under the condition that average pay-off for both strategies are sufficiently similar, or alternatively, in which larger agglomerations of players choosing the same strategy exist for both strategies. In this case, clusters of size 9 exists for both strategies after the initial interaction period. An unbalanced initial distribution is the limit situation, in which the entire player population chooses the same strategy except for one minimal mutant cluster of size 6. Alternatively, it illustrates a situation, in which players choose their strategy at random, but average pay-offs are very different, so that the player population collapses into large clusters of the risk dominant strategy and small clusters of playing the risk inferior strategy with at least one being of size 6. Both distributions are the possible extreme cases that will define the boundary conditions for the evolution of a stable convention.

From Ille 2013 we have again the following proposition

Proposition 3. A population with balanced initial distribution converges to the pay-off superior equilibrium $h_{B}$, if the pay-off premium $\rho$ is greater than $\frac{1}{7}(a-c)$. If the pay-off premium is smaller, but positive, a player population consists of clusters playing different strategies.

Figure 3 shows the result of a set of simulations identical to those in figure 2 , but given $d=4, b=2 c=a-2$ and $a$ ranging from 3.6 to 4.4. Furthermore, each strategy is initially played by $50 \%$ of the population and seeding is completely random. Thresholds are again as expected. The population converges to equilibrium $(B, B)$ for $a$ smaller than 3.6 and to $(A, A)$ for values larger or equal to 4.3. The population thus converges to the Pareto optimal convention, except if the pay-off premium is within a marginal perceptible unitd

Abandoning the assumption that both conventions are risk equivalent, allows us, in this context, to study the effect of risk and its relation to efficiency. As before,

$\mathrm{d} \frac{1}{7}(a-c)$ defines the marginal perceptible unit, under which no pure equilibrium will occur. Furthermore, the simulation shows that for a small number of periods, the distribution is affected by the relative average pay-off, but stabilizes after the initial interaction period. 
Fig. 3. Percentage of strategy $A$ players; $\mathrm{d}=4, \mathrm{~b}=2, \mathrm{c}=\mathrm{a}-2, a \in(3.6,4.4 ; 0.1) t \in(1,50)$

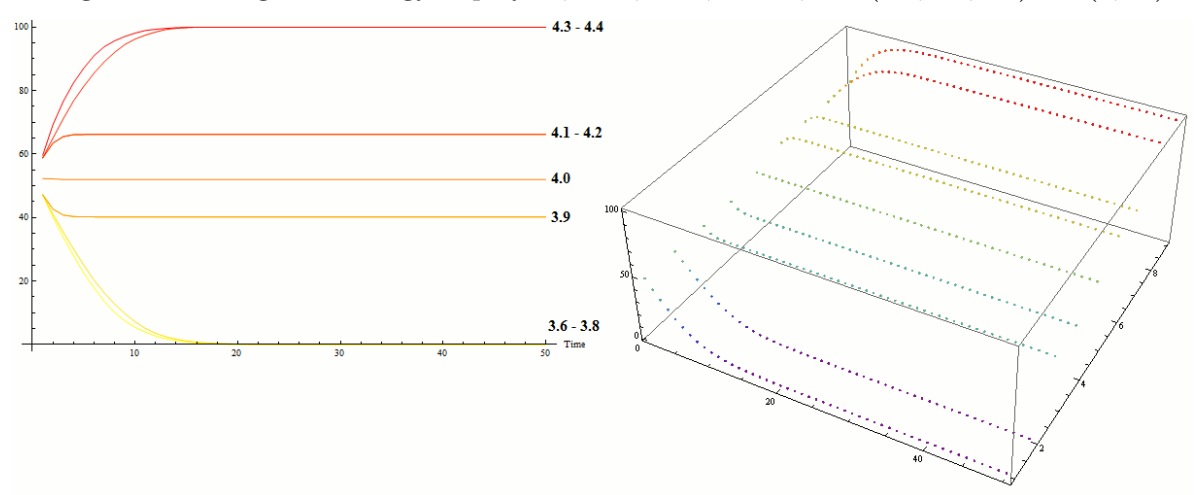

we assume that $d=a+\rho$ and $b=c+\rho$. In addition, we integrate a risk premium $\mu$ by substituting $c$ in matrix 1 with $\hat{c}$, such that $\hat{c}=c-\mu$. In Ille 2013 we obtained that

Proposition 4. Given a coordination game as in matrix (1) with two equilibria of which $h_{B}$ pay-off dominates $h_{A}$ by a pay-off premium of $\rho$, and $h_{A}$ risk dominates $h_{B}$ by a risk premium of $\mu$, the population converges to convention $h_{B}$ if

$$
\mu< \begin{cases}c-a+7 \rho, & \text { and the initial distribution is balanced } \\ c-a+\frac{5}{3} \rho, & \text { and the initial distribution is at least unbalanced }\end{cases}
$$

If the initial population distribution is unbalanced and $\mu>\frac{2(c-a)+4 \rho}{3}$, the population chooses the risk dominant convention. In the case that a population is, however, initially sufficiently balanced, the risk dominant strategy only prevails as a convention, if it also pay-off dominates by a value greater than $\frac{a-c}{7}$. Otherwise the population remains in a state of mixed conventions.

Fig. 4. Percentage of strategy $A$ players; $d=4, b=3, \hat{c}=2, a=[3.2,3.3 ; 0.01] t \in(1,30)$

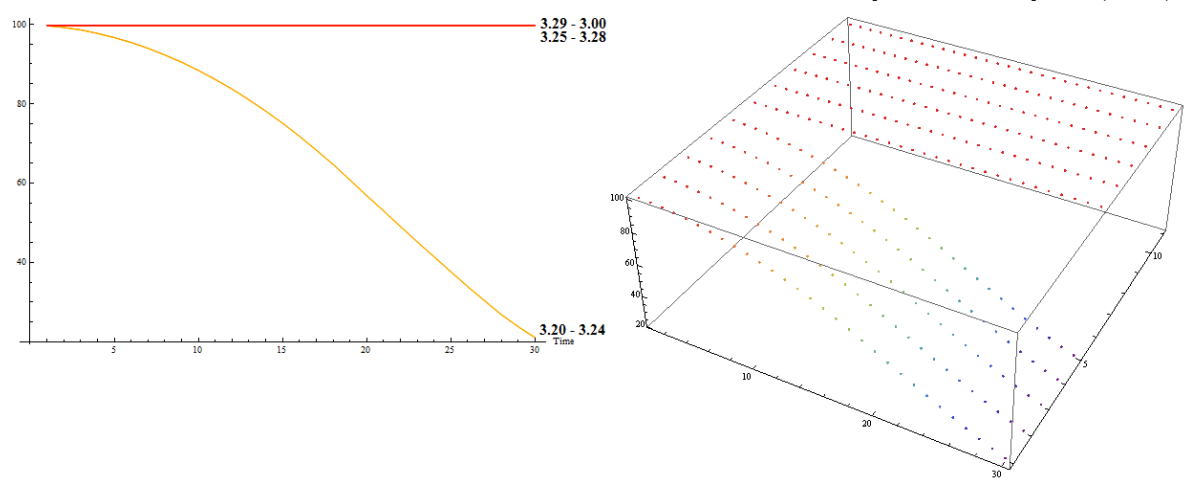


Figure 4 shows the result of a set of simulations identical to those in figure 2 i.e. given an unbalanced initial distribution, but $\hat{c}$ is fixed at $\hat{c}=2$ and $a$ ranges from 3.0 to 3.3. The population converges to the Pareto dominant equilibrium for values of $a$ smaller and equal to 3.24 and converges to the risk dominant equilibrium for $a>3.29$. For $\frac{1}{4}<\mu<\frac{2}{7}$, the population converges to a mixed equilibrium, with a few square shaped clusters of size 6 or 9 that play the Pareto dominant strategy.

Fig. 5. Percentage of strategy $A$ players; $d=4, b=2, \hat{c}=1, a \in(3.0,4.0 ; 0.1) t \in(1,15)$

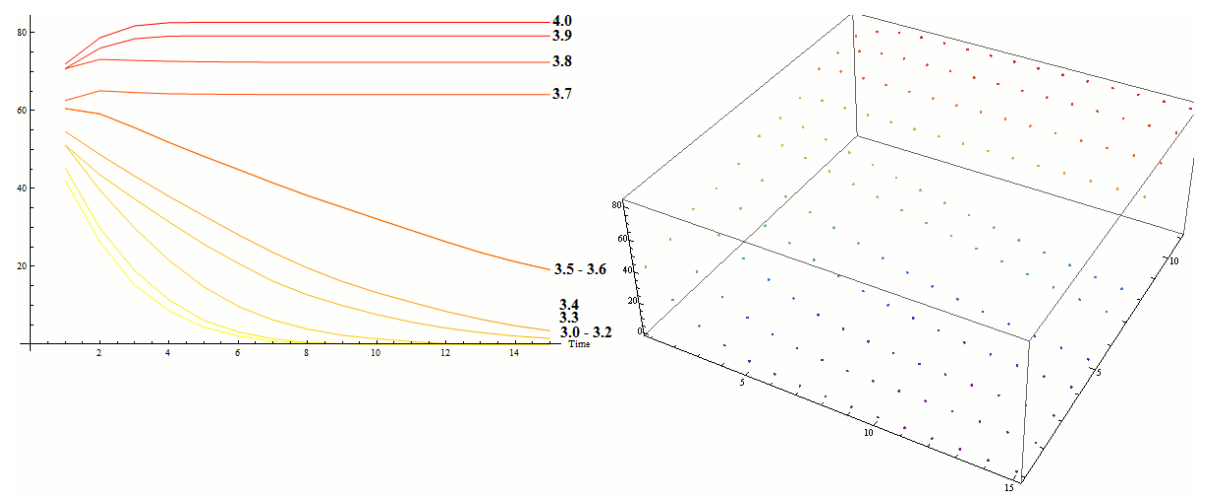

Figure 5 presents the result of a set of simulations identical to those in figure 3 i.e. given a balanced initial distribution, but $\hat{c}=1$ and $a$ ranges from 3.0 to 4.0. The population converges to the Pareto dominant equilibrium for values of $a$ smaller and equal to 3.6 and remains in a mixed equilibrium for larger values.

Fig. 6. Percentage of strategy $A$ players; $a \in(0,20 ; 1)$, Pay-off Premium $(-10,10 ; 1)$ and zero risk premium

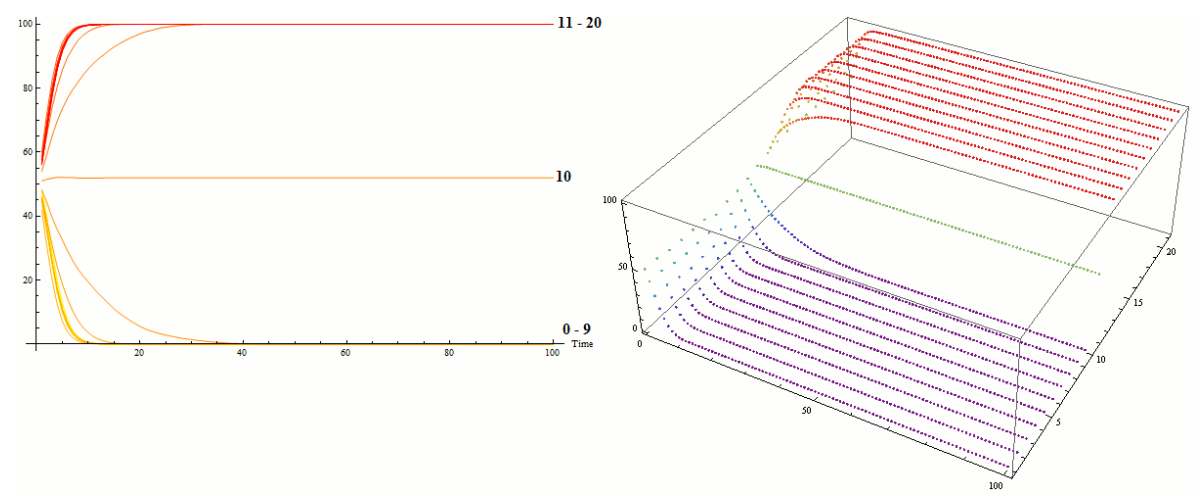

Figure 6 shows the distribution of the player population for $t \in(0 ; 100)$ and a changing pay-off premium $\rho$. The pay-off structure is given by matrix 1 , where 
$b=6, d=10, \hat{c}=a-4$ and $a$ takes values from 0 to 20 in unit steps. The effect of the size of $\rho$ on convergence speed towards a single equilibrium is negligible. The same holds for the updating probability.

Fig. 7. Percentage of strategy $A$ players; Convergence for different updating probabilities: Probability $(1,100 ; 9)$

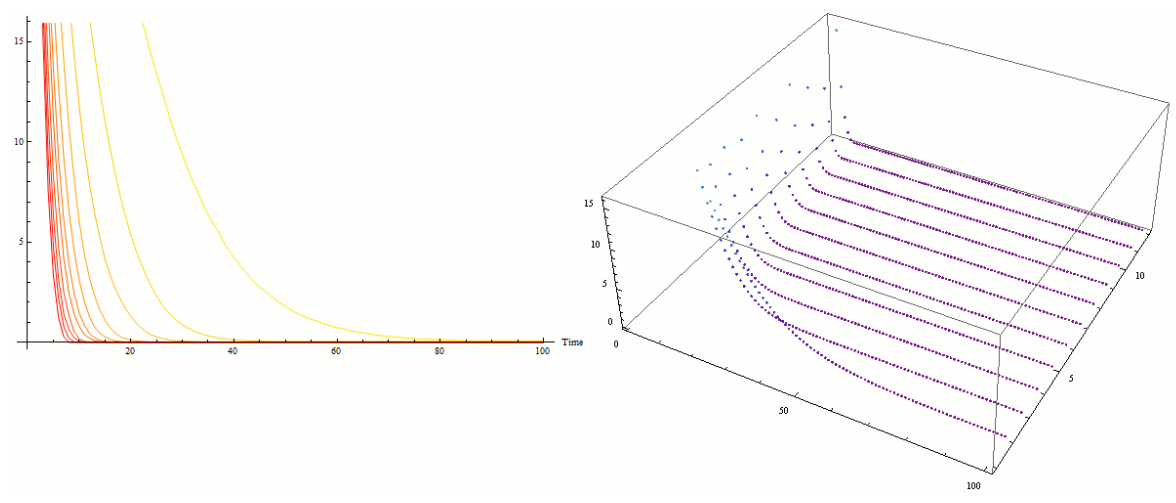

Differences in the updating rule has no impact on the final outcome, but only on the time required for a transition. Figure 7 shows the distribution for pay-offs $a=3, b=2, \hat{c}=1$ and $d=4$ and different updating probabilities. The updating probability ranges from 1 to 100 in steps of 9 .

\section{General $2 \times 2$ Coordination Game}

The following section analyzes the dynamics of general $2 \times 2$ coordination games, ceteris paribus, in which two player types (row and column) interact with each other. The general pay-off structure is defined by the following pay-off matrix:

$$
\begin{aligned}
& \text { A B } \\
& \begin{array}{l}
A \\
B
\end{array}\left(\begin{array}{cc}
a_{1}, a_{2} & b_{1}, \hat{c}_{2} \\
\hat{c}_{1}, b_{2} & d_{1}, d_{2}
\end{array}\right)
\end{aligned}
$$

Define as before $\rho_{i}=b_{i}-c_{i}$ or $\rho_{i}=d_{i}-a_{i}$ and hence $\hat{c}_{i}=c_{i}-\mu_{i}$, for $i=1,2$. It must also hold that $a_{i}>\hat{c}_{i}$ and $d_{i}>b_{i}$.

The fundamental dynamics are defined by only a few conditions similar to what has been obtained for the single type case. Only $2 \times 6$ conditions have to be analyzed in the general game. To derive this and as a first step, remember that the conditions for the pay-off structure, in addition to the imitation principle and the local interaction generate three useful characteristics.

(1) In general, the player choosing the Pareto dominant strategy with respect to his type benefits from the relative abundance of players in his neighborhood that 
belong to the other player type and are playing the same strategy. If $a_{i}>b_{i}$ and $d_{i}>\hat{c}_{i}, \forall i=1,2$, this also holds for the strategy that is not Pareto dominant.

(2) A strategy change will only occur at the edges of clusters. This property must also hold for mixed clusters, i.e. if both player types play different strategies on the same patches in a neighborhood.

(3) After the initial period of interaction, the strategy distribution on the grid will be determined by the relative average pay-off of each strategy, since it is more likely that a player adopts the strategy that has a higher average pay-off if players initially choose their strategy at random with equal probability.

Subsequent to a transition period, large uniform clusters with cluster size 9 (for most clusters) occur after an initial period of interaction. Each pushes towards a convention and the "strongest push" will eventually prevail. This is, however, only the case if the average pay-off for both strategies are not too different and the distribution is initially sufficiently balanced. Even if players choose a strategy at random with equal probability before the first interaction, too diverse average payoffs will inhibit the evolution of clusters - constituted by players of the risk inferior strategy that have sufficient size to overtake the player population. In general, the edges of clusters will be either horizontal, vertical or diagonal. Consequently, the clusters' shape can be generalized to the following three types:

Fig. 8. The three variants of cluster edges - numbers indicate the number of players with the same strategy in the individuals neighborhood, cluster are supposed to continue beyond the figure's frame. (Figure taken from Ille 2013].)

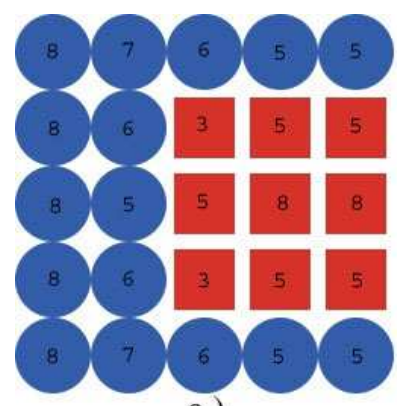

a.)

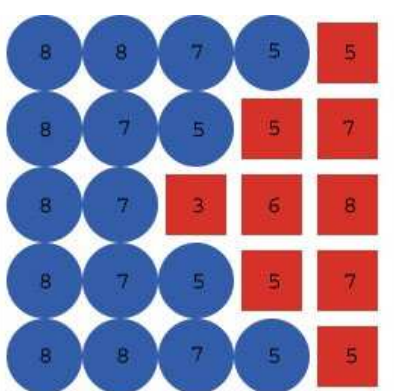

b.)

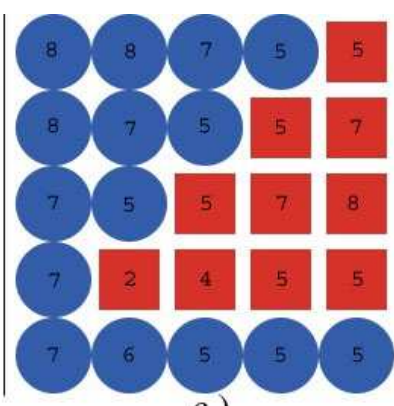

C.)

From this figure we observe that, though various parameter combinations could lead to strategic changes, only six conditions for each strategy influence the dynam- 
ics of the entire population in the long-term:

\begin{tabular}{|c|c|c|}
\hline I. & $\begin{array}{l}\text { For } s_{i}=A \text { to overtake } s_{i}=B \\
\eta_{A}=1: \quad 7 a_{i}+b_{i}>8 d_{i}\end{array}$ & $\begin{array}{l}\text { For } s_{i}=B \text { to overtake } s_{i}=A \\
\eta_{B}=1: \quad 7 d_{i}+\hat{c}_{i}>8 a_{i}\end{array}$ \\
\hline II. & $\eta_{A}=2: \quad 6 a_{i}+2 b_{i}>8 d_{i}$ & $6 d_{i}+2 \hat{c}_{i}>8 a_{i}$ \\
\hline III. & $5 a_{i}+3 b_{i}>8 d_{i}$ & $5 d_{i}+3 \hat{c}_{i}>8 a_{i}$ \\
\hline IV. & $4 a_{i}+4 b_{i}>8 d_{i}$ & $4 d_{i}+4 \hat{c}_{i}>8 a_{i}$ \\
\hline $\mathrm{V}$ & $3 a_{i}+5 b_{i}>8 d_{i}$ & $3 d_{i}+5 \hat{c}_{i}>8 a_{i}$ \\
\hline VI. & $\eta_{A}=6: \quad 2 a_{i}+6 b_{i}>8 d_{i}$ & $\eta_{B}=6: \quad 2 d_{i}+6 \hat{c}_{i}>8 a_{i}$ \\
\hline
\end{tabular}

for $i=1,2$. The condition I. and III. are identical to those found in proposition 4 for the single type case. The first condition implies that clusters can be overtaken along diagonal edges. It will turn an inlying cluster (red) as in a.) into an inlying cluster as in b.). If both conditions in I. are fulfilled, i.e. one for each player type, these corner elements will continuously switch between strategies. Condition III. applies to the horizontal and vertical cluster edges. It will not affect the players surrounding the corner elements of inlying clusters (see b.) and c.) ). Hence, an inlying quadrangular cluster will expand and will incrementally turn the horizontal or vertical edge into a diagonal edge. Since condition III. includes condition I., the cluster will also continue to expand along these diagonal edges. Furthermore, under condition III. any inlying cluster can be invaded. The remaining conditions have a minor effect on the convergence speed than the aforementioned. Condition IV. and above only concern the growth along the corner elements.

The easiest asymmetric game is a game of "common interest". This denotes a pay-off structure, in which the same strategy is Pareto dominant for both types. According to the former notation, either $a_{i}>d_{i}$ for both $i=1$ and $i=2$ (or the inverse). If at least one player type fulfills at least the first condition given by $\eta_{A}=1$ $\left(\eta_{B}=1\right)$, the population either converges to the convention defined by $h_{A}\left(h_{B}\right)$, or ends up in a mixed equilibrium with interior rectangular shaped clusters playing $A$ that cannot expand (see 8.a.). This depends on the initial random distribution and the average pay-off of each strategy. Note that condition I. is in this case exactly the same as in proposition 4 , given the balanced initial distribution. If at least one type meets condition $\eta_{A}=3\left(\eta_{B}=3\right)$, the convention is surely defined by $h_{A}$ $\left(h_{B}\right)$. Since both player types either prefer equilibrium $(\mathrm{A}, \mathrm{A})$ or equilibrium $(\mathrm{B}, \mathrm{B})$, the convergence by one player type towards an equilibrium is not counter-acted by the other player type. Convergence speed is irrelevant for the final distribution and convention, but the more conditions are fulfilled by one or both types, the faster the population converges to its Pareto dominant equilibrium.

If individuals find themselves in a "conflict game", in which the Pareto dominant equilibria are not identical, the convention is defined by the equations in 3 . The strategy that fulfills the higher condition in 3 will define the convention. In the following, I will simulate a player population to confirm that population dynamics behave according to the previous results.

The most convenient way to test for the correctness of these results is to fix pay- 
off parameters of one player type at the different levels at which the constraints in 3 can be fulfilled, e.g. ranging from none to all six. The dynamics for each parameter of the other player type are then simulated with respect to each of these levels. As a basis for the analysis, assume the following pay-off matrix:

A

B

$$
\begin{gathered}
A \\
B
\end{gathered}\left(\begin{array}{cc}
a_{1}=3.5, a_{2}=3.5 & b_{1}=3, \hat{c}_{2}=2 \\
\hat{c}_{1}=2, b_{2}=3 & d_{1}=4, d_{2}=()
\end{array}\right)
$$

In order to account for the various variables, at which the conditions can be fulfilled, $d_{2}$ is set to one of 6 different values, namely $d_{2}=3.16 ; 3.22 ; 3.28 ; 3.34 ; 3.41 ; 3.47$ in each simulation run. This implies values of convergence speed given by $\eta_{A}=5 ; 4 ; 3 ; 2 ; 1 ; 0$ and that player type 2 converges to $h_{A}=(A, A)$ except for $d_{2}=3.47$ and thus $\eta_{A}=0$. At the last parameter value, player type 2 does not exhibit any convergence.

For each of these values, one parameter of player type 1 is analyzed by a set of simulations. In the first set of simulations the parameters of player type 2 are set to the values in 4 and $d_{2}=3.16$. For the first simulation of this set, one parameter of player type 1 is fixed at its lowest value, at which it does not fulfill any condition (or all, depending on the parameter) of the first column in 3. His other pay-off parameters are set to the values as in matrix 4 (if not stated otherwise). The initial distribution is set to 50 : 50 (if not stated otherwise), with completely random seeding. After the system has been simulated for a fixed number of periods, the parameter of player type 1 is changed by an increment and the system is simulated again. Using the same initial distribution renders the results directly comparable. Simulations are repeated until the parameter reaches a maximum value, at which all condition (or none) of the first column in 3 are fulfilled. Hence, player type 1 will progressively converge to equilibrium $(B, B)$, whereas player type 2 will converge to equilibrium $(A, A)$ at the speed determined by the value of $d_{2}$. After the value of player 1's parameter has reached its maximum value, the set of simulations is repeated for each of the remaining values of $d_{2}$ and at the beginning of each set of simulations the population is "seeded" anew. Each remaining parameter of player type 1 is analyzed in the same way, obtaining 6 sets of simulations for each parameter of player type 1 , thus 24 sets of simulations in total. The figures show the proportion of type 1 players choosing strategy $A$. Since the distribution for both types concurs after the initial periods, it suffices to graph one player type, as before.

Figure 9 shows the result for parameter $a_{1}$. In order to maintain the assumption that $a_{i}>b_{i}$, the value of $b_{1}$ is adapted accordingly and set to $b_{1}=2.3$. This change is made only for the simulations concerning $a_{1}$. $a_{1}$ takes value from 2.375 to 3.875 in increments of 0.25 . If both types have the same convergence speed, the population converges to a mixed equilibrium, where the strategy distribution is determined during the initial periods of interaction, i.e. by the average pay-off and the random initial distribution. In order to compensate this effect (since for $\rho_{A}=\rho_{B}, a_{1}$ is 
Fig. 9. Simulation for $a_{1} \in(2.375,3.875 ; 0.25)$ a.) $\eta_{A}=5$; b.) $\eta_{A}=4$; c.) $\eta_{A}=3$; d.) $\eta_{A}=2$; e.) $\eta_{A}=1$; f.) $\eta_{A}=0$; in c.)-f.) initial share of $B_{0}=55 \%$

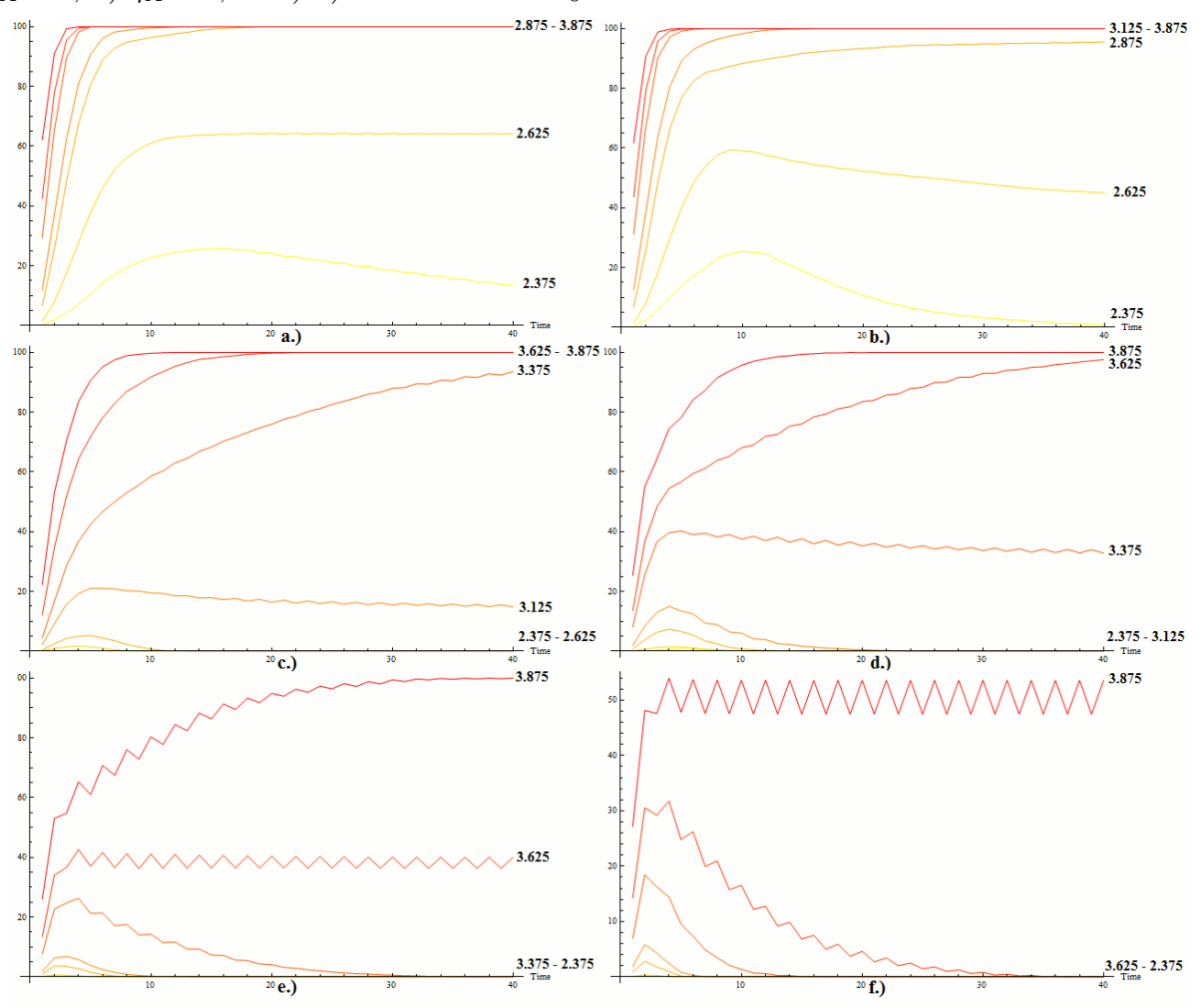

relatively large in the later simulations), the initial distribution was set to $55 \%$ strategy $B$ players in the last 4 simulations. The predicted threshold values from the equations in 3 for the parameters are as shown in table 1.

Table 1. Convergence speed for each player type in the simulations

\begin{tabular}{|l|c|c|c|c|c|c|c|}
\hline \multicolumn{8}{l}{$\eta_{A}<\eta_{B} \rightarrow h_{B}=(B, B), \eta_{A}>\eta_{B} \rightarrow h_{A}=(A, A)$} \\
\hline$\eta_{A}$ & 0 & 1 & 2 & 3 & 4 & 5 & \\
\hline$d_{2}$ & 3.47 & 3.41 & 3.34 & 3.28 & 3.22 & 3.16 & \\
$\eta_{B}$ & 0 & 1 & 2 & 3 & 4 & 5 & 6 \\
\hline$a_{1}<$ & 4 & 3.75 & 3.5 & 3.25 & 3 & 2.75 & 2.5 \\
$c_{1}>$ & & 0 & 2 & $\frac{8}{3}$ & 3 & 3.2 & $\frac{10}{3}$ \\
$d_{1}>$ & 3.5 & $\frac{26}{7}$ & 4 & 4.4 & 5 & 6 & 8 \\
\hline
\end{tabular}


Figure 10 on page 14 shows the results for parameter $b_{1}$. It takes value from -4 to 3 in increments of 1 . Looking at 3 shows that $b_{1}$ is extraneous in the constrains of the right column. $b_{1}$ only affects the average pay-off and thus the number of strategy $A$ players after the initial sequence of interactions. It thereby has an indirect impact on the time required to converge to an equilibrium, since it modifies the initial sequence after which the distribution of convergence to the convention. $\eta_{B}=1$ satisfies $8 a_{1}=2 c_{1}^{*}+6 d_{1}$ only as equality and it only holds that $8 a_{1}<\hat{c}_{1}+7 d_{1}$. Consequently, convergence to $(B, B)$ is only observable for $d_{2}=3.47$. For $d=3.41$ the convergence speed for both player types is identical and thus stable mixed equilibria occur. In the case of $d=3.34$ convergence to $(A, A)$ is slow as condition II. is met with equality by player type 2 . Similarly the simulation for $b_{1}=3$ and $d=3.47$ approaches $(B, B)$ slowly as strategy $A$ players is frequent after the initial sequence of interactions.

Fig. 10. Simulation for $b_{1} \in(-4,3 ; 1)$ a.) $\eta_{A}=5$; b.) $\eta_{A}=4$; c.) $\eta_{A}=3$; d.) $\eta_{A}=2$; e.) $\eta_{A}=1$; f.) $\eta_{A}=0$

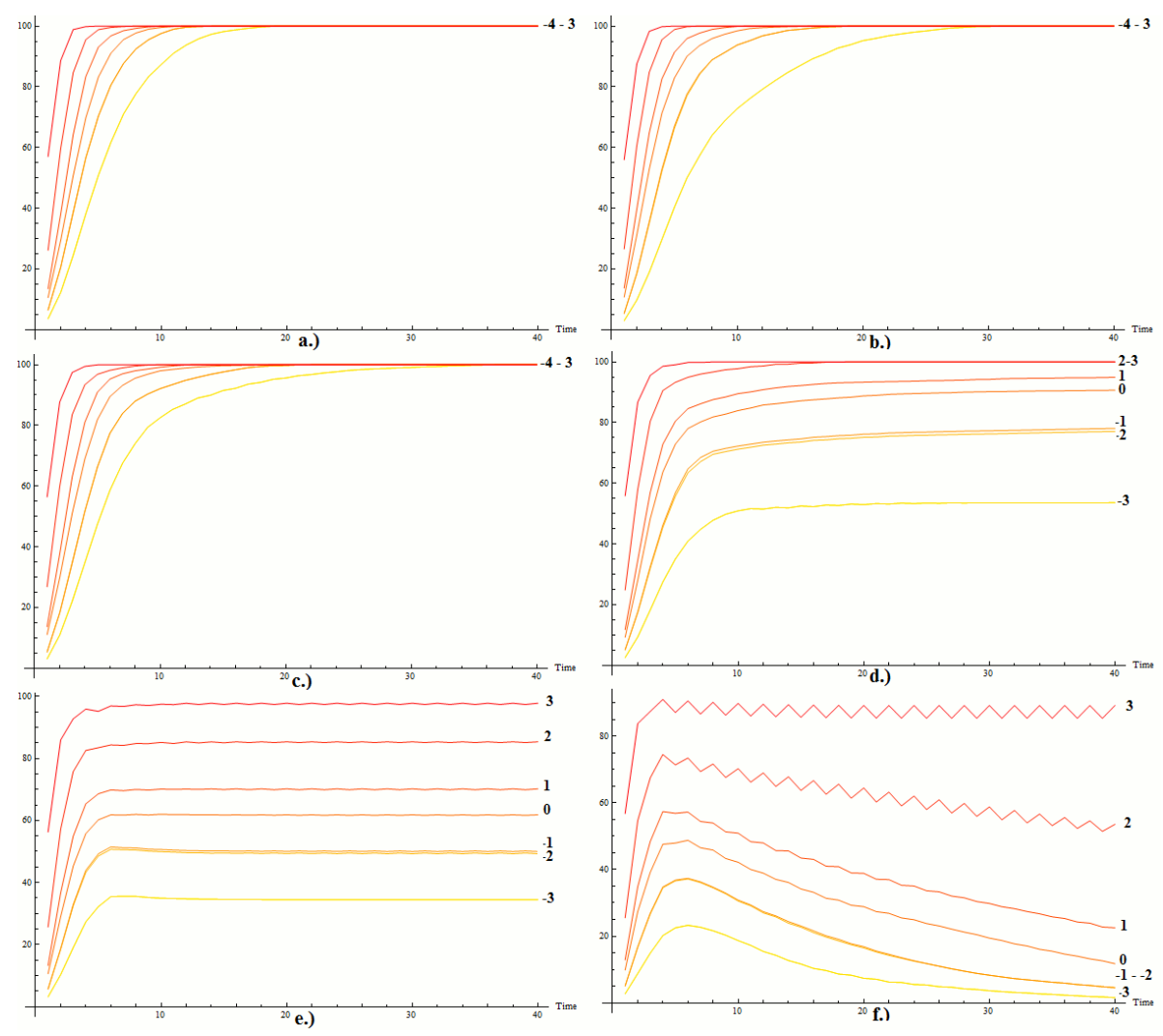

$\hat{c}_{1}$ adopts value from -0.1 to 3.5 in increments of 0.2 in the simulations in figure 
11 on page 15. Since $c_{1}$ can take small critical values, simulations are conducted with an initial distribution of $55 \%$ strategy $B$ players in the first two simulations and $60 \%$ strategy $B$ players in the later simulations, in order to avoid that the cluster size of strategy $B$ is too small after the initial sequence of interactions. The threshold values for this parameter are again to be found in table 1 .

Fig. 11. Simulation for $\hat{c}_{1} \in(-0.1,3.5 ; 0.2)$ a.) $\eta_{A}=5$; b.) $\eta_{A}=4$; c.) $\eta_{A}=3$; d.) $\eta_{A}=2$; e. $)$ $\eta_{A}=1$; f.) $\eta_{A}=0$; a.)-b.) $55 \%$ strategy $B$ players, c.)-f.) $60 \%$ strategy $B$ players

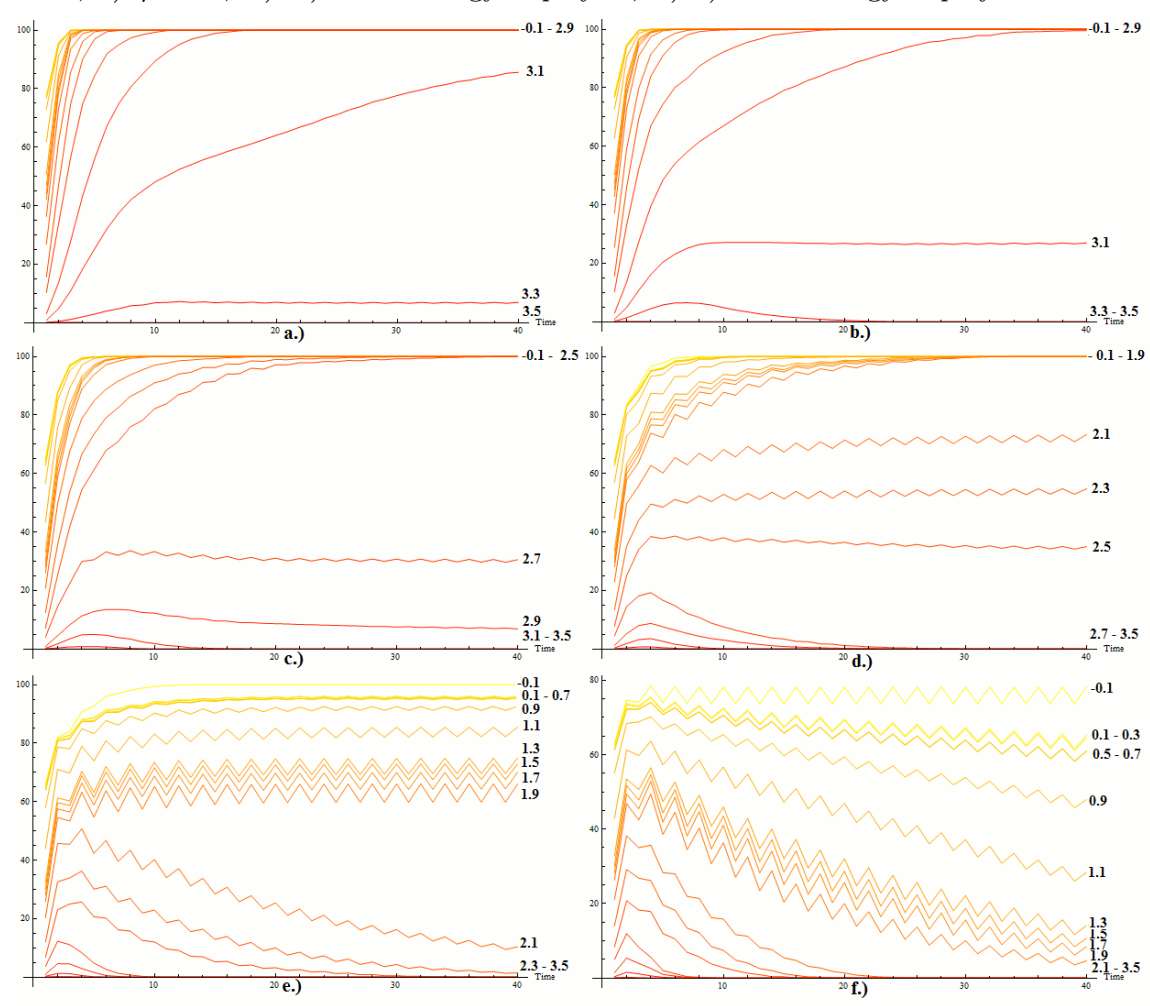

The final set of figures 12 on page 16 shows the dynamics for $d_{1} \cdot d_{1}$ takes values from 3.2 to 8.48 in increments of 0.33 . In order to compensate for the "average pay-off effect" the share of initial strategy $B$ player was set to $60 \%$ in the last 4 simulations.

All simulations behaved as in table 1 . The population can converge to different equilibria, though the level of risk dominance and the level of Pareto dominance are equal. Figure 13 shows a set of simulations for $b_{1}=3, \hat{c}_{1}=1, a_{2}=4, b_{2}=1, \hat{c}_{2}=3$ and $d_{2}=3 . a_{1} \in(2.0,7.0 ; 0.1)$ and $d_{1}=a_{1}+1$. Hence, for all simulations $|\rho|=1$ and $|\mu|=1$, and strategy $A$ is risk superior and strategy $B$ Pareto superior for type 1 , and the inverse for type 2 . Note that by the equations in 3 the conditions, determining the dynamics, are unaffected by linear transformations of the pay-offs. 
Fig. 12. Simulation for $d_{1} \in(3.2,8.48 ; 0.33)$ a.) $\eta_{A}=5$; b. $) \eta_{A}=4$; c.) $\eta_{A}=3$; d.) $\eta_{A}=2$; e. $)$ $\eta_{A}=1$; f.) $\eta_{A}=0$; c.)-f.) $60 \%$ strategy $B$ players

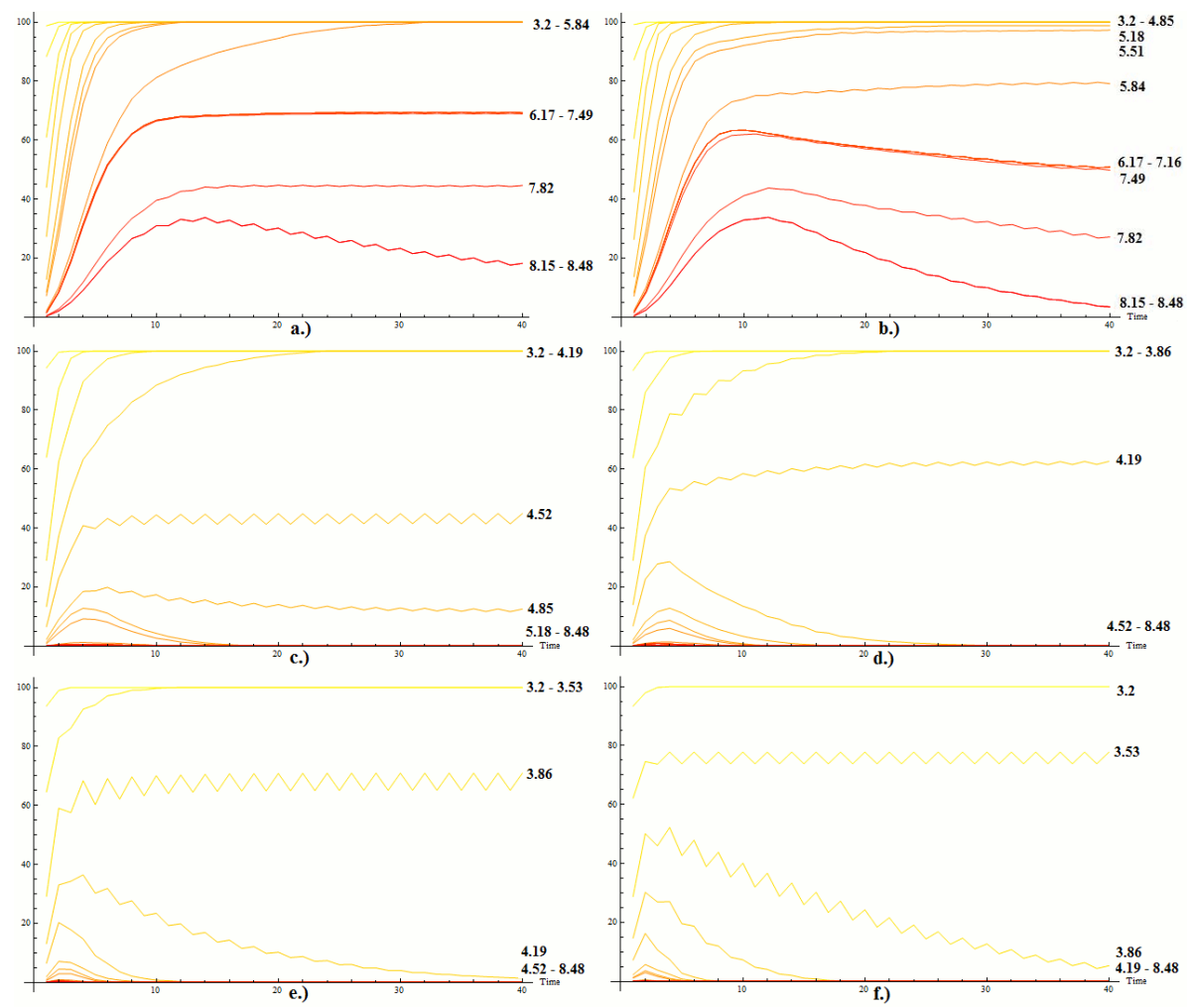

In addition, such a transformation will have no effect on the relative average pay-off.

\section{Conclusion}

This paper has replicated the analytical results of Ille 2013 and, on the basis of simulations, has provided a clearer understanding of the dynamics during the transition periods. In the spatial context of $2 \times 2$ coordination game with imitations, a population converges either to one of the pure Nash equilibria or a mixed conventional state, in which the population consists of large clusters of players that either play the Pareto or risk dominant strategy. The likelihood of the evolution of a certain convention is defined by a non-linear relationship between pay-off (Pareto) dominance and risk dominance. This implies that if conventions illustrate the same risk levels ${ }^{\text {e }}$, the more efficient evolves on the long-term. This tendency can only be offset if the other convention offers a sufficiently large risk premium. We thus

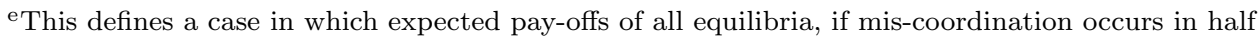
of the cases, are identical. 
Fig. 13. Percentage of strategy $A$ players; Set of simulations with $a_{1} \in(2.0,7.0 ; 0.1)$ and $d_{1}=a_{1}+1$ : $|\rho|=1$ and $|\mu|=1$

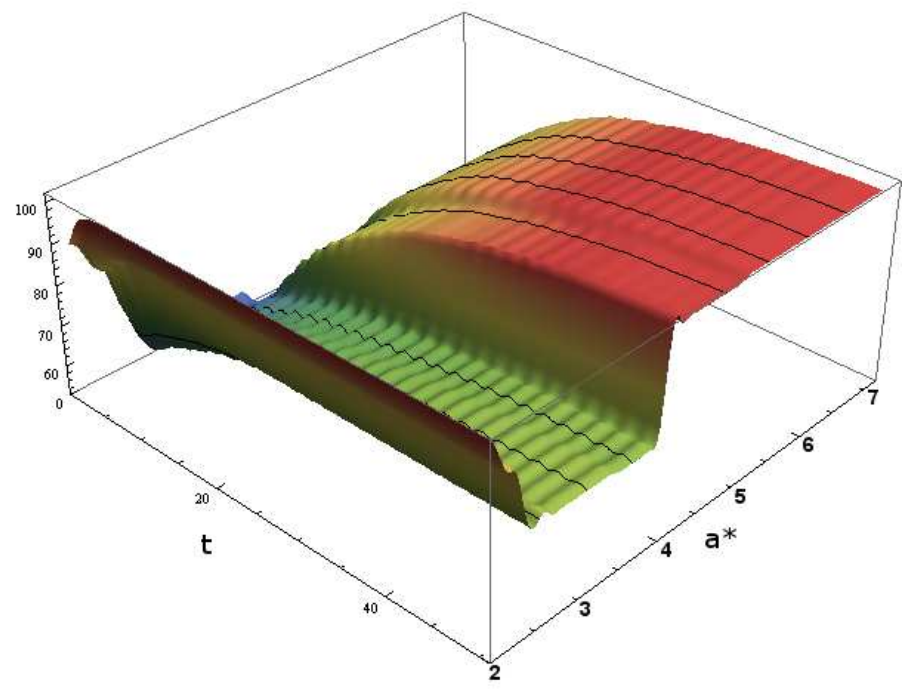

observe that risk dominance is a rather weak indicator of the long-term equilibrium if it is considered detached from pay-off efficiency. In fact, if a conventional behavior does not exist a priori and has to evolve in a population, either a population converges to the Pareto dominant convention or, if both conventions offer almost equal pay-off, the population will be in a stable state, in which both conventions co-exist.

In the case, in which two player populations interact, each population might consider another convention as pay-off dominant. In this conflict game, the equilibrium, which offers the highest pay-off premium to one of the populations, defines the long-term convention. Populations thus illustrate a tendency to depart from egalitarian conventions and highly unequal conventions evolve after some time.

Some possible extensions for further research have already been discussed in Ille 2013. In respect to simulations, the extension to more than two strategies and a broader number of games is of interest. Preliminary simulations show a number of spatial patterns that can evolve and are thus worthy of a more detailed analysis $f$

${ }^{\mathrm{f}}$ First simulations of an extended Prisoner's Dilemma with three strategies, in which the defective strategy can exploit the two others, and a semi-defective strategy can only exploit the cooperative strategy, shows very interesting patterns. A structure evolves that is similar to a class society, in which the middle class works as a buffer between the other two. 


\section{References}

Bicchieri, Cristina [2006] The Grammar of Society: The Nature and Dynamics of Social Norms. Cambridge University Press.

Bowles, Samuel [2006] Microeconomics: Behavior, Institutions, and Evolution (The Roundtable Series in Behavioral Economics). Princeton University Press.

Hodgson, Geoffrey F. [1996] Economics and Evolution: Bringing Life Back into Economics. University of Michigan Press.

Huntington, Samuel P. and Harrison, Lawrence E. [2004] Streit um Werte: Wie Kulturen den Fortschritt prgen. Goldmann Verlag.

Ille, Sebastian [2013] The dynamics of norms and convention under local interactions and imitation. International Game Theory Review (under review).

Nowak, Martin A. and May, Robert M. [1992] Evolutionary games and spatial chaos. Nature, 359, 826-829.

Page, Scott E. [2007] The Difference: How the Power of Diversity Creates Better Groups, Firms, Schools, and Societies. Princeton University Press. 